\title{
Bio-signal based elbow angle and torque simultaneous prediction during isokinetic contraction
}

\author{
ZHOU Yu ${ }^{1}$, LIU Jingbiao ${ }^{1}$, ZENG Jia ${ }^{1}$, LI Kairu² \& LIU Honghai ${ }^{2 *}$ \\ ${ }^{1}$ State Key Laboratory of Mechanical System and Vibration, School of Mechanical Engineering, \\ Shanghai Jiao Tong University, Shanghai, 200240, China. \\ ${ }^{2}$ The School of Computing, The University of Portsmouth, Portsmouth, PO1 3HE, UK
}

\begin{abstract}
It is of great importance to decode motion dynamics of the human limbs such as the joint angle and torque in order to improve the functionality and provide more intuitive control in human-machine collaborative systems. In order to achieve feasible prediction, both the surface electromyography (sEMG) and A-mode ultrasound were applied to detect muscle deformation and motor intent. Six abled subjects were recruited to perform five trails elbow isokinetic flexion and extension, and each trail contained five repetitions, with muscle deformation and sEMG signals recorded simultaneously. The experimental datasets were categorized as: the ultrasound-EMG combined datasets, ultrasound-only datasets and EMG-only datasets. The support vector machine (SVM) regression model was developed for both elbow joint angle and torque prediction, based on the above three kinds of datasets. The root-mean-square error (RMSE) and the correlation coefficients (R) were applied to evaluate the prediction accuracy. The results across all the subjects for different datasets indicated that the combined datasets and the ultrasound datasets were superior to the sEMG datasets both on elbow joint angle and torque prediction, and there were no significant differences between the combined datasets and the ultrasound datasets. It turns out that elbow angle and torque can be reconstructed by Amode ultrasound, and the significant findings pave the way towards the application of musculature-driven human-machine collaborative systems.
\end{abstract}

Angle, torque, surface electromyography (sEMG), ultrasound, support vector machine (SVM), regression, isokinetic contraction.

\section{Introduction}

Understanding the characteristics of human physiological signals and proposing corresponding autonomous and adaptive learning methods for accurate perception of human behaviour play an essential role in realizing the tri-co robot natural interactions [1]. One of the bottleneck problems is how to adapt human motion, such as torque etc, for human-machine collaborative systems, covering a wide spectrum of human-centred applications primarily on prosthesis control and rehabilitation systems. The study gives priority to the challenging problem, that is to say, simultaneous prediction of human elbow joint angle and torque for musculature-driven human-machine systems.

*Corresponding author (email: honghai.liu@port.ac.uk)
The dominant biomedical signal employed in musculature-driven systems is electromyography (EMG) though very recently emergent biomedical signals also be considered. EMG is generally recorded in two protocols: invasive electromyography (iEMG) and surface electromyography (sEMG). iEMG, with a needle or fine-wire inserted to the muscle of interest, shows high spatial selectivity and can be applied to identify individual motor unit (MU) electrical activities. However, the risk of infection and pain caused by iEMG acquisition make it rejective across users. Hence, sEMG, detected by the non-invasive surface electrodes on skin, can overcome some of the limitations of iEMG and has been more widely applied in prostheses control [2], robots control [3] and human-machine interfaces [4]. The motor intent can be detected from sEMG signals during muscle contraction to control external equipment [5]. Researches have focused on pattern classification-based techniques, in which 
the sEMG patterns are classified into several discrete classes of motor intents, to improve the functionality and provide more intuitive control of myoelectric prostheses and other devices [6]-[8]. To provide simultaneous control, recent work has investigated the use of regression techniques to relate sEMG to a continuous motor variable such as force or position [9][10].

Sonomyography (SMG), representing the muscle deformation detected by ultrasound with high spatial and temporal resolution, is one of the most commonly used diagnostic tools to assess the functions of skeletal muscles in both research studies and clinical applications [11]-[13]. Some studies focused on the application of extracting features of SMG. Castellini et al. extracted the spatial gradient features based on the uniformly-spaced grid of interest points among the ultrasound images to predict the finger positions and found a clear linear relationship between the features and the finger positions as well as the fingertip forces [14], [15]. Feature-based discrete finger movements or hand motions classification using A-mode ultrasound [16]-[20] or B-mode ultrasound [21] showed very good results. The B-mode ultrasound is expensive and bulky in general, while the A-mode transducers can be made into smaller size and be easier attached to the skin during dynamic movements. So, the A-mode ultrasound can be a more portable human-machine interface solution, compared with B-mode ultrasound.

Isokinetic dynamometry is a well-accepted tool for assessing strength of the upper and lower extremities, and ioskinetic testing is an useful approach to assess limb extension and flexion [22][23]. The peak torque can be detected continuously by the isokinetic device during ioskinetic movement, when the related muscles are on maximum voluntary contraction (MVC). Thus, the muscle-contraction degree differences among people can be eliminated.

In the aspect of utilized machine-learning algorithms, the support vector machine (SVM) has been widely used in EMG-related pattern recognition [24] and it showed good performance. It also has been applied using B-model ultrasound to predict wrist angle [25] and joint angle [26]. Besides, Guo et al. has proved that SVM models show better performance in predicting wrist angle based on A-mode ultrasound than ANN models [27]. Ameri et al. has verified that SVMbased regression outperformed ANN-based method in realtime and simultaneous myoelectric control [9]. Therefore, we applied SVM regression model to decode the elbow angle and torque from the upper arm bio-signals.

Based on the understandings above all, we hypothesized that the upper arm muscle deformation and sEMG can be used to decode the elbow angle and elbow torque for prosthesis and robotic arm control. The system frame for the application is shown in Figure 1. The aims of this pilot study are: 1) examining the feasibility of applying the bio-signals to elbow angle as well as torque simultaneous decoding and prediction ; 2) exploring the optimal bio-signals datasets for elbow torque decoding and prediction. The rest paper is organized as follow. The Section 2 presents the methods and experiments. The experimental results are presented in Section 3. Finally, Section 4 concludes this study with discussions and future work.

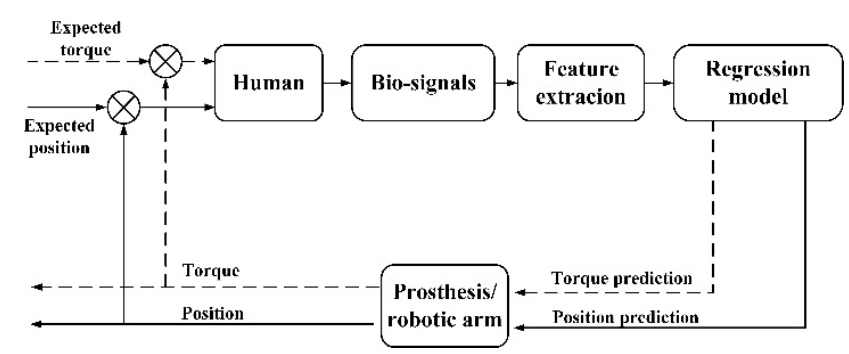

Figure 1 Framework of human-machine interface based on the positon and torque decoding.

\section{Material and methods}

\subsection{Subjects}

Six healthy male subjects (aged 22-27; referenced as S1-S6) were invited to participate to the experiment. None of them has any history of neuromuscular disorder and each has been given a written informed consent prior to the experiment. It should be noted that all the experiment procedures in this study have been approved by the Shanghai Jiao Tong University’s Mechanical Engineering Ethics Committee.

\subsection{Experimental protocols}

The experimental protocols are described as follow: the subjects seat comfortably in the adjustable chair of the Isomed2000 isokinetic device (D\&R Ferstl GmbH, Hemau, Germany) as shown in Figure 2. One's body was fixed by a

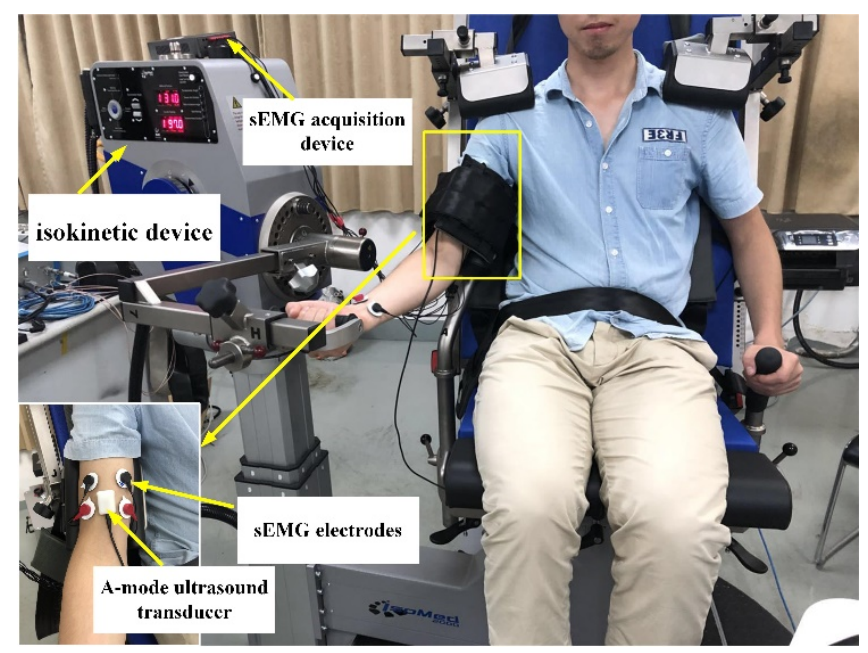

Figure 2 Experimental setup for the measurement of muscle ioskinetic contraction with A-mode ultrasound and sEMG. 


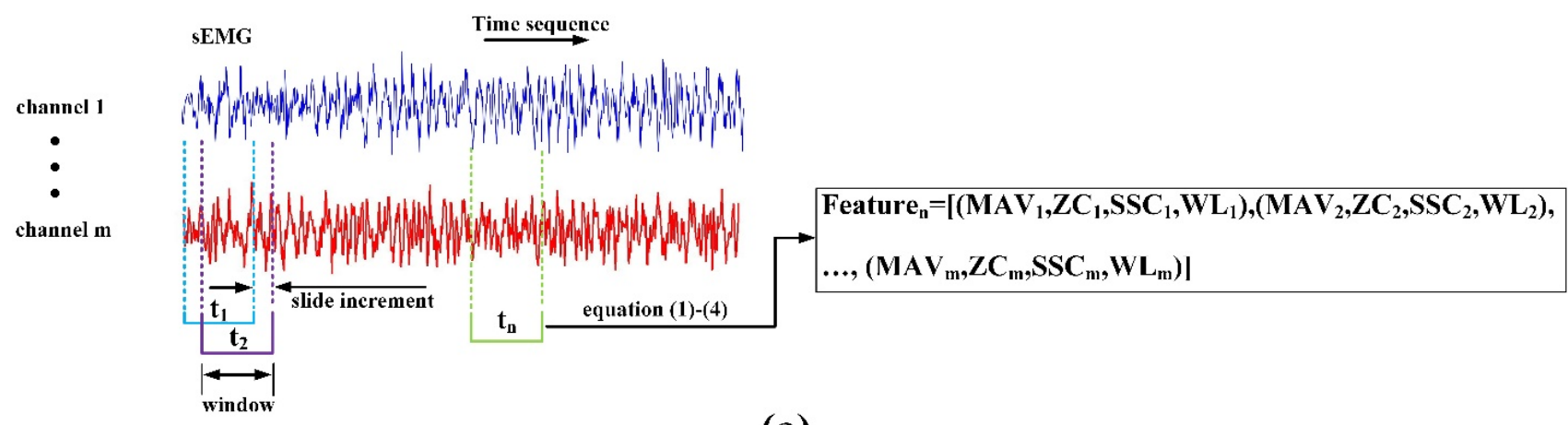

(a)

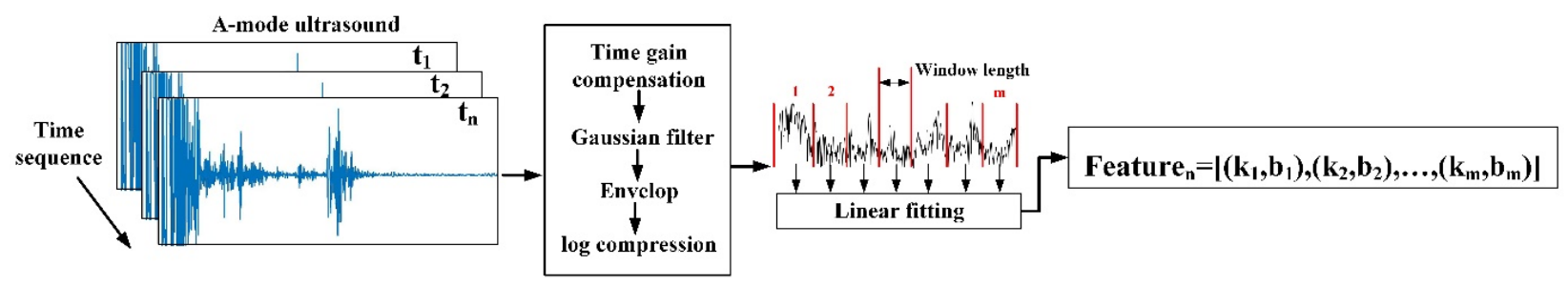

(b)

Figure 3 The flowchart of feature extraction for sEMG and ultrasound: (a) multi-channel sEMG feature extraction at time tn, $\mathrm{m}$ denotes the channel number; (b) ultrasound signal feature extraction at time tn, m denotes the window number. Featuren denotes the generated feature sets at time tn.

belt and one's shoulders fixed by two girdles onto the chair back to stabilize the posture during the experiments. The right upper arm was placed and fixed on the holder. The wireless multi-channel sEMG acquisition device (Jiaopu Tech Ltd, China) was applied to capture the sEMG signals. The signals were processed with band-pass filtered (pass band 20-500 $\mathrm{Hz}$ ), sampled at $1000 \mathrm{~Hz}$, and A/D converted with 12-bit resolution. The commercial multi-channel A-mode transducer driver board (Zhongxu Tech Ltd, China) was applied to drive the $5 \mathrm{MHz}$ single element ultrasound transducers (diameter: $14 \mathrm{~mm}$, height: $18 \mathrm{~mm}$ ), and to amplify and digitized the received signals. Correlative study in [28] had proved that 5 $\mathrm{MHz}$ was the optimum frequency with a good SNR in both low and high frequency domains. The frame rate and sampling rate were set as $20 \mathrm{~Hz}$ and $100 \mathrm{MHz}$ respectively. In each frame, 8192 data dots were sampled and stored. Since the sound velocity in human tissues is around $1540 \mathrm{~m} / \mathrm{s}$, the detection depth of the system through human tissue can reach about $63.1 \mathrm{~mm}$. Thus, the related deep muscles' deformation in upper arm can be detected. The two channels bipolar electrodes (diameter: 25mm) of EMG acquisition device were placed on the belly of musculus biceps brachii muscle parallel to the muscle fiber direction, and another two channels bipolar electrodes were placed on the belly of musculus triceps brachii muscle. One channel A-mode ultrasound transducer was placed on musculus biceps brachii muscle belly, with standard ultrasound gel applied between the transducer and the skin.

After all the sensors configuration, the Isomed2000 were set to isokinetic-test mode and the subjects held the isokinetic dynamometer to perform elbow extension and flexion at a speed of 60 degree/second, and the elbow extension and flexion movements were repeated for five times continuously for one trial, with sEMG, A-mode ultrasound, elbow angle and elbow joint torque synchronized and recorded simultaneously. Five trials was performed in total in the same subject with one minute for rest between each trial to avoid muscle fatigue. It should be noted that the experiment would be stopped once subjects report any uncomfortable feeling.

\section{3 sEMG feature extraction}

The classical EMG time domain (TD) features were extracted and made up the four-dimensional EMG feature set corresponding to one channel. The TD features were namely, mean absolute value (MAV), zero crossings (ZC), slope sign changes (SSC), and waveform length (WL), which were calculated by the equation (1), equation (2), equation (3) and equation (4) respectively. The raw EMG data were segmented into a series of 300 ms windows with an overlap of $250 \mathrm{~ms}$, and features were extracted from these windows. The multi-channel sEMG feature extraction method is shown in Figure 3(a).

$$
\begin{gathered}
\mathrm{MAV}=\frac{1}{n} \sum_{i=1}^{n}\left|x_{i}\right| \\
\mathrm{ZC}=\sum_{i=2}^{n} \operatorname{sgn}\left(-x_{i} x_{i-1}\right)
\end{gathered}
$$




$$
\begin{gathered}
\mathrm{SSC}=\sum_{i=2}^{n-1} \operatorname{sgn}\left[\left(x_{i}-x_{i-1}\right)\left(x_{i}-x_{i+1}\right)\right] \\
\mathrm{WL}=\sum_{i=2}^{n}\left|x_{i}-x_{i-1}\right|
\end{gathered}
$$

where $n$ is the window size and $x_{i}$ is the EMG signals.

\subsection{Ultrasound feature extraction}

The feature extraction method for A-mode ultrasound signals has been used in our previous study [17] [18] and is similar to the mean image gradient feature extraction applied in Bmode ultrasound to predict finger positions [14]. As shown in Figure 3(b), The raw A-mode ultrasound signal of each frame was processed by time gain compensation, Gaussian filtering, envelop and log compression to improve the signalto-noise rate [29]. Then the processed data was segmented by a fixed window. In this study, there were 8192 data dots sampled by the driver board in each frame, in which the first 292 data dots and the last 100 data dots were discarded; and the window length was set as 100 data dots. Thus, there were 78 segments corresponding to each frame for further processing. The linear fitting was applied to each segment to get the slope "k" and intercept "b". All the slopes and intercepts detected from the 78 segments were combined together to make up the feature vector for each frame. Finally, the elbow angle/torque and the corresponding A-mode ultrasound feature vectors were combined together to form the data sets for SVM regression and prediction.

\subsection{The regression model}

In this study, the SVM regression model was developed and trained to predict the forearm position and elbow torque with different datasets as inputs. The basic SVM is to map the input data $x$ from the inseparable low dimensional feature space to a high linearly separable dimensional feature space:

$$
\mathrm{y}=\omega^{T} \varphi(x)+b
$$

where $\varphi$ is a nonlinear mapping function, and $\omega$ and $b$ are the weight vector and bias term respectively. The SVM regression can be described as the equality constraint:

$$
\min _{\omega, b, e} J_{P}(\omega, e)=\frac{1}{2} \omega^{T} \omega+\gamma \frac{1}{2} \sum_{k=1}^{N} e_{k}^{2}
$$

such that

$$
y_{k}=\omega^{T} \varphi\left(x_{k}\right)+b+e_{k}, k=1, \cdots, N
$$

where $\gamma$ is the weight in determining the balance between minimizing the training errors and finding the optimal hyperplane and $e_{k}$ are the slack variables. The Lagrange multiplier method was used to solve the optimization problem above:

$$
\begin{aligned}
& L(\omega, b, e ; \alpha)=J_{P}(\omega, e)-\sum_{k=1}^{N} \alpha_{k} \omega^{T} \varphi\left(x_{k}\right)+b+e_{k}- \\
& y_{k}
\end{aligned}
$$

where $\alpha_{k}$ are the Lagrange multipliers. After setting the partial derivatives for $\omega, b, e_{k}$ and $\alpha_{k}$ to zero, an linear equation set is obtained:

$$
\begin{gathered}
{\left[\begin{array}{cc}
0 & 1_{v}^{T} \\
1_{v} & \delta+I / \gamma
\end{array}\right]\left[\begin{array}{l}
b \\
\alpha
\end{array}\right]=\left[\begin{array}{l}
0 \\
y
\end{array}\right]} \\
\delta=\varphi\left(x_{k}\right)^{T} \varphi\left(x_{l}\right)=K\left(x_{k}, x_{l}\right), k, l=1, \ldots, N
\end{gathered}
$$

where $1_{V}$ is the $\mathrm{N}$-dimensional vector $[1, \ldots, 1]^{T}, I$ is a $\mathrm{N}$ dimensional unit vector and $\alpha=\left[\alpha_{1}, \ldots, \alpha_{N}\right] . K\left(x_{k}, x_{l}\right)$ is the kernel function. The SVM regression model is defined as follow:

$$
\mathrm{y}(\mathrm{x})=\sum_{k=1}^{N} \alpha_{k} K\left(x, x_{k}\right)+b
$$

where $K\left(x, x_{k}\right)$ is the Gaussian radial basis Kernel function (RBF-Kernel) [30]. The offline estimation accuracy was determined using a five-fold estimation cross validation, where four out of five trails of experimental data for every subject were included in the training set and the remaining one trial data were used in the test set (80\% training, 20\% testing).

Accuracy evaluation of the elbow angle and torque predictions from different datasets was made by calculating the root- mean-square error (RMSE) and the correlation coefficients $(\mathrm{R})$ of the measured and predicted values. Generally speaking, if relative RMSE $(\%)<15 \%$ and R $>0.9$, the prediction is regarded as excellent [31]. The RMSE and R are defined by equation (12) and equation (13).

$$
\text { RMSE }=\sqrt{\frac{\sum_{n=1}^{N}(X(n)-Y(n))^{2}}{N}}
$$

$$
\mathrm{R}=\frac{\sum_{n=1}^{N}(X(n)-\bar{X}(n))(Y(n)-\bar{Y}(n))}{\sqrt{\sum_{n=1}^{N}(X(n)-\bar{X}(N))^{2} \sum_{n=1}^{N}(Y(n)-\bar{Y}(n))^{2}}}
$$

where $X(n)$ is the true value, $Y(n)$ is the predicted value. $\bar{X}(n)$ and $\overline{Y(n)}$ are the means of $X(n)$ and $Y(n)$ respectively. $N$ is the number of data points.

\subsection{Statistical analysis}

The one-way ANOVA was applied in IBM SPSS Statistics (IBM Inc, USA) to compare the regression accuracy resulting from EMG-only, ultrasound-only and combined EMGultrasound on RMSE and R, based on the data of all subjects. The level of statistical significance was set at $p<0.05$ for all statistics. 


\section{Experimental results}

\subsection{Root-mean-square error}

The results of the RMSE as shown in Figure 4 (a) and (b) are for the angle prediction and torque prediction based on different datasets. As for the elbow angle prediction, the RMSE ranged from $8.25 \% \pm 1.23 \%$ (S3) to $13.03 \% \pm 4.43 \%$ (S4) for the ultrasound-EMG combined datasets, $8.94 \% \pm$
$1.30 \%$ (S3) to $14.74 \% \pm 1.44 \%$ (S5) for the ultrasound datasets and $14.92 \% \pm 2.75 \%$ (S6) to $24.66 \% \pm 4.05 \%$ (S2) for the EMG datasets, as inputs respectively; the mean values were $10.35 \% \pm 2.10 \%, 11.52 \% \pm 2.47 \%$ and $19.07 \% \pm 3.41 \%$ corresponding to the above three kinds of datasets in order across all subjects. As for the elbow torque prediction, the corresponding RMSE ranged from $7.92 \% \pm 1.31 \%$ (S5) to $11.54 \% \pm 3.35 \%$ (S2), $8.73 \% \pm 1.79 \%$ (S6) to $14.38 \% \pm 2.18 \%$ (S4) and $10.08 \% \pm 2.02 \%$ (S5) to $15.97 \% \pm 3.05 \%$ (S2),
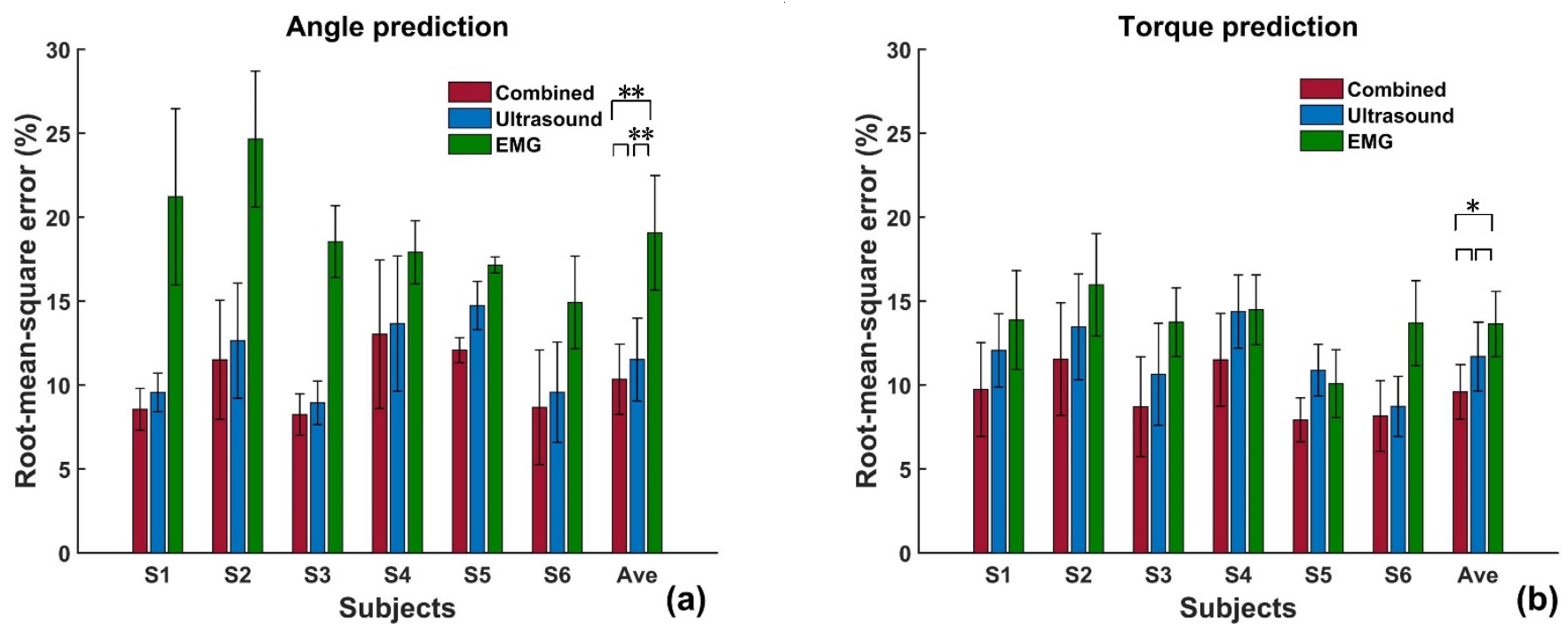

Figure 4 The regression accuracy comparison of RMSE between actual and predicted value on elbow angle and torque : (a) the result of angle prediction; (b) the result of torque prediction. Ave denotes the average value of all subjects, error bars represents the stand deviation. One asterisk “*” denotes $p<0.05$, and two asterisks “**” denote $p<0.001$.
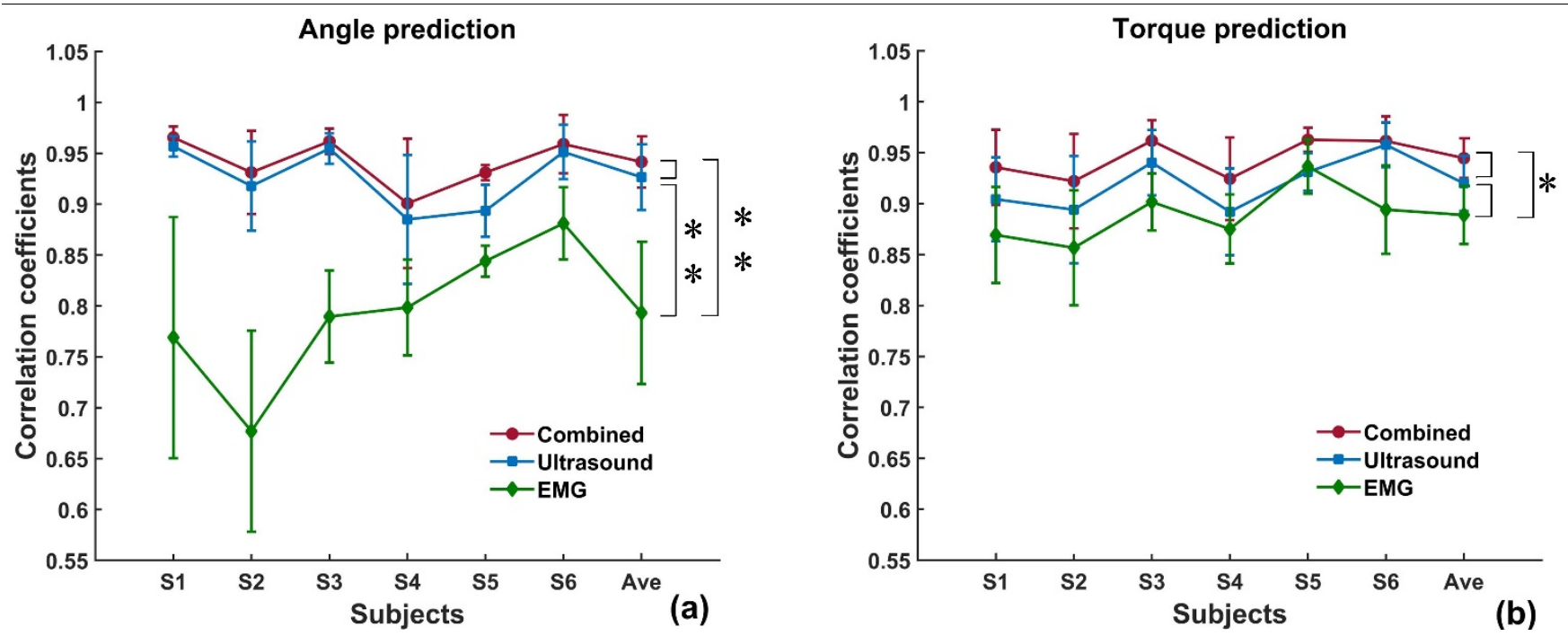

Figure 5 The regression accuracy comparison of $\mathrm{R}$ value between actual and predicted value on elbow angle and torque : (a) the result of angle prediction; (b) the result of torque prediction. Ave denotes the average value of all subjects, error bars represents the stand deviation. One asterisk “ $*$ ” denotes $p<0.05$, and two asterisks “**” denote $p<0.001$. 

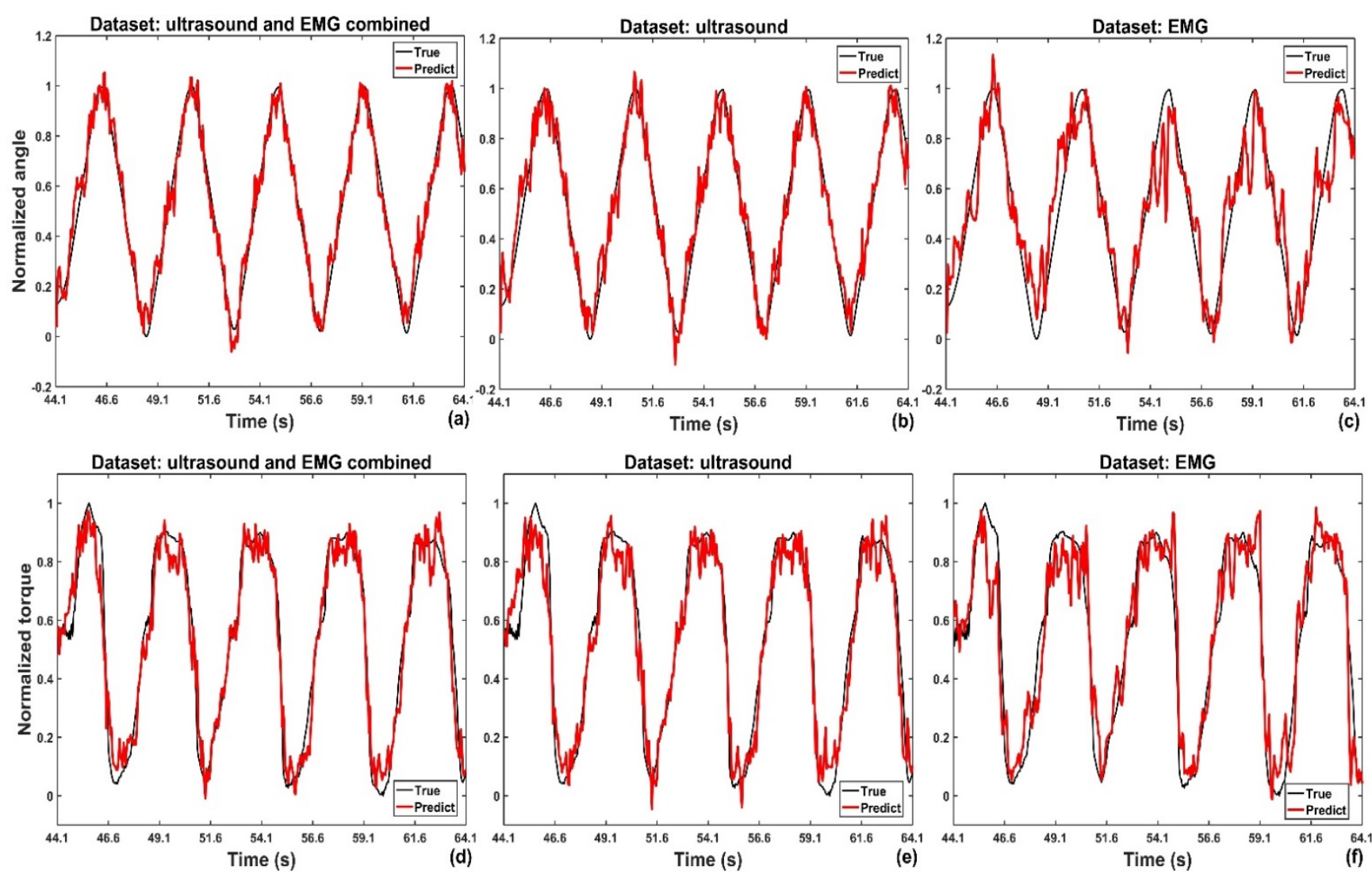

Figure 6 Typical demonstrations of comparisons between true and predicted elbow angle and torque for subject S6: (a) angle prediction based on the combined dataset; (b) angle prediction based on the ultrasound dataset; (c) angle prediction based on the EMG dataset; (d) torque prediction based on the combined dataset; (e) torque prediction based on the ultrasound dataset; (d) torque prediction based on the EMG dataset.

and the mean values were $9.60 \% \pm 1.62 \%$ for the ultrasoundEMG combined datasets, $11.70 \% \pm 2.05 \%$ for the ultrasound datasets and $13.64 \% \pm 1.94 \%$ for the EMG datasets, as inputs respectively. It was found that the combined datasets showed the best performance both on elbow angle prediction and elbow torque prediction. The ultrasound datasets showed excellent performance because the mean RMSE $<15 \%$ for angle prediction and torque prediction. The EMG datasets were of the worst performance among the three datasets, especially for the angle prediction (the mean RMSE $>15 \%$ ).

The ANOVA analysis indicated that there were significant improvements in angle prediction accuracy when using the ultrasound-EMG combined datasets or the ultrasound datasets as inputs, compared with the EMG inputs; the differences were not significant between the combined datasets and ultrasound datasets for the angle prediction ( $p=0.466$ ). As for the torque prediction, the significant improvements took place when using combined datasets, compared with the EMG datasets, as regression model inputs; however, there were no significant differences between the combined datasets and the ultrasound datasets ( $p=0.072$ ), and between the ultrasound datasets and the EMG datasets $(p=0.093)$.

\subsection{Correlation coefficients}

The results of the $\mathrm{R}$ value comparison for angle prediction and torque prediction is shown in Figure 5 (a) and (b). As for the elbow angle prediction, the $\mathrm{R}$ value ranged from $0.901 \pm$ 0.064 (S4) to $0.965 \pm 0.011$ (S1) , $0.885 \pm 0.063$ (S4) to 0.957 \pm 0.010 (S1) and $0.677 \pm 0.099$ (S2) to $0.881 \pm 0.035$ (S6) when using the ultrasound-EMG combined datasets, ultrasound datasets and EMG datasets as inputs respectively; the corresponding mean values were, namely, $0.942 \pm 0.025$, $0.927 \pm 0.032$ and $0.793 \pm 0.070$. As for the elbow torque prediction, the $\mathrm{R}$ value ranged from $0.922 \pm 0.046$ (S2) to $0.963 \pm 0.012$ (S5) , $0.892 \pm 0.043$ (S4) to $0.958 \pm 0.022$ (S6) and $0.857 \pm 0.056$ (S2) to $0.936 \pm 0.027$ (S5), and the mean values were $0.945 \pm 0.020$ for the ultrasound-EMG combined datasets, $0.920 \pm 0.027$ for the ultrasound datasets and $0.889 \pm 0.029$ corresponding to the EMG datasets across all the subjects, respectively. The results further verified that the combined datasets outperformed the ultrasound datasets and the EMG datasets. The ultrasound datasets also showed 

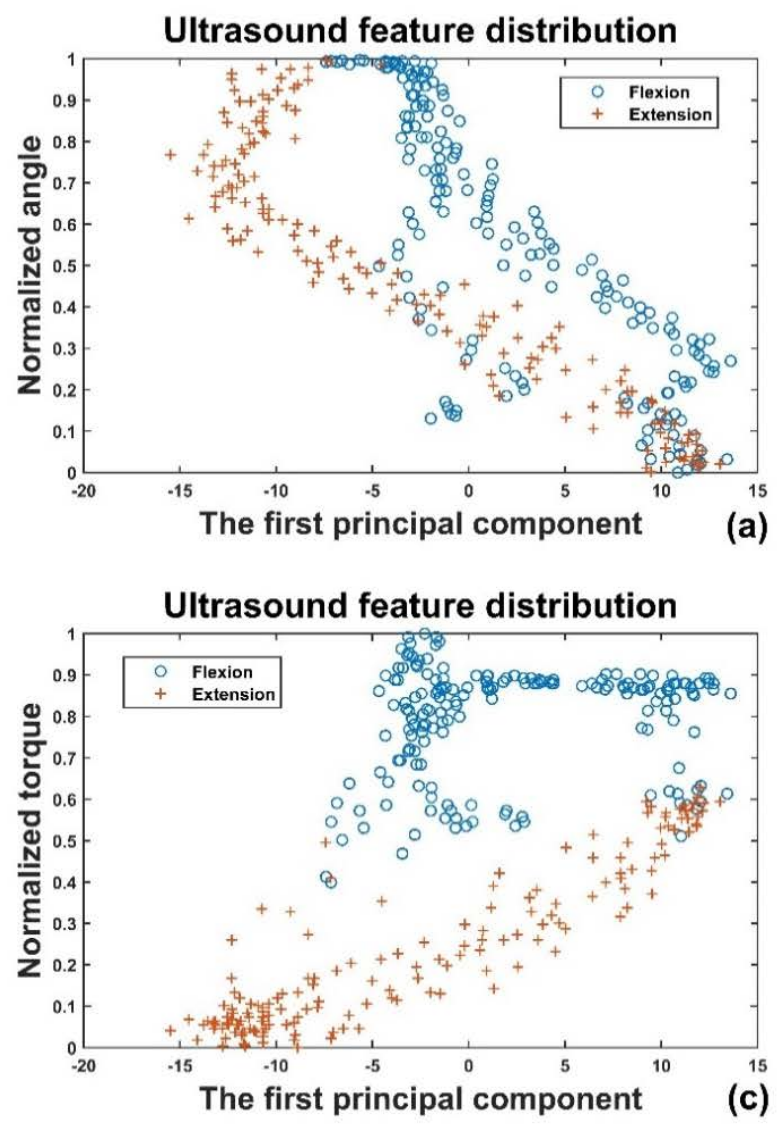
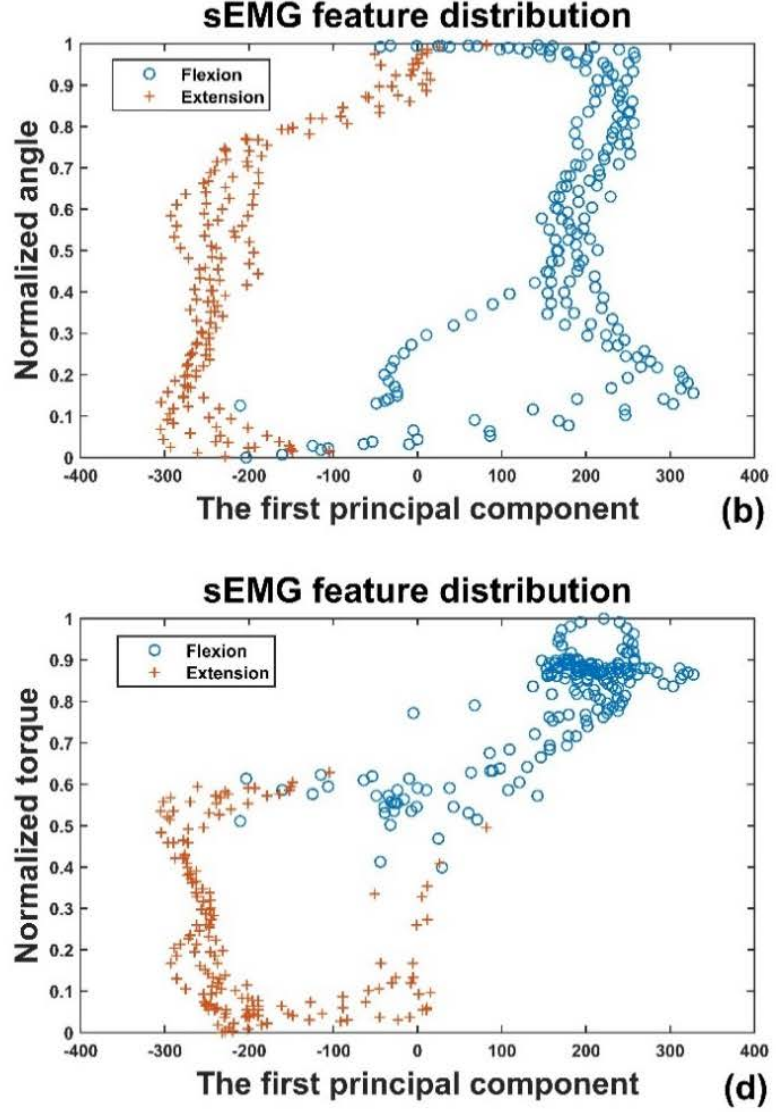

Figure 7 The feature distribution of ultrasound and sEMG signals, corresponding to the first principal component and elbow angle/torque. To make it clear, the features are presented in different label, in terms of randomly selected five cycles continuous flexion and extension processes of subject S6: (a) ultrasound signal feature distribution respect to elbow angle ; (b) sEMG signal feature distribution respect to elbow angle; (c) ultrasound signal feature distribution respect to torque; (d) sEMG signal feature distribution respect to torque.

excellent performance with mean $\mathrm{R}>0.9$ for angle prediction as well as torque prediction. The EMG datasets were of the worst performance among the three datasets, especially for the angle prediction (the mean $\mathrm{R}<0.8$ ).

The ANOVA analysis showed the consistent results as in RMSE analysis. The significant improvements took place in angle prediction when using the ultrasound-EMG combined datasets or the ultrasound datasets as inputs, compared with the EMG inputs; the differences were not significant between the combined datasets and ultrasound datasets $(p=0.586)$. As for the torque prediction, the significant improvements only happened between the combined datasets and the EMG datasets; the differences were not significant between the combined datasets and ultrasound datasets $(p=0.111)$, and between the ultrasound datasets and EMG datasets ( $p=0.051)$.

\section{Discussion and conclusion}

In this study, we captured the sEMG signals and ultrasound signals from the upper arm during the elbow isokinetic flexion and extension, which excluded the muscle force differences during dynamic muscle contraction among people, making the regression and prediction of elbow angle and torque evaluation more objective. The muscle deformation was detected by one channel A-mode ultrasound and the electrophysiological information was represented by four channels sEMG signals. The EMG datasets, ultrasound datasets and EMG-ultrasound combined datasets were applied to train the SVM regression model respectively to predict the elbow angle and torque simultaneously. The combined datasets outperformed the other two datasets across all subjects ( $\mathrm{RMSE}=10.35 \%$ and $\mathrm{R}=0.942$ for elbow angle prediction; $\mathrm{RMSE}=9.60 \%$ and $\mathrm{R}=0.945$ for elbow torque prediction). Besides, the ultrasound datasets (RMSE $=11.52 \%$ and $\mathrm{R}=0.927$ for elbow angle prediction; $\mathrm{RMSE}=11.70 \%$ and $\mathrm{R}=0.920$ for elbow torque prediction) were superior to the EMG datasets $(\mathrm{RMSE}=19.07 \%$ and $\mathrm{R}=0.793$ for elbow angle prediction; $\mathrm{RMSE}=13.64 \%$ and $\mathrm{R}=0.889$ for elbow torque prediction). The results clearly suggested that the upper arm muscle deformation could be used to reconstruct the elbow 
angle and torque simultaneously based on the SVM model.

\subsection{Optimal bio-signal datasets}

Figure 6 shows the typical comparisons between true and predicted elbow angle/torque based on the data of subject S6, which demonstrates the effect of different datasets vividly. Obviously, the four channels EMG signal datasets were not qualified for the prediction, especially for the elbow angle evaluation. And to improve the performance, more channels EMG signals should be involved.

Both the ultrasound-EMG combined datasets and the ultrasound datasets performed excellently, and the combined datasets outperformed the ultrasound datasets according to Figure 4 and 5. However, the differences were not significant between the combined datasets and the ultrasound-only datasets according to the ANOVA analysis. Besides, the EMG signals can be easily contaminated by ambient noise, which would decrease the performance of the combined datasets. Hence, considering the system complexity and wearability, the one channel A-mode ultrasound is more acceptable for potential prosthetics or robot control application. On the one hand, the study in [14] had verified the B-mode ultrasound features can be used to decode joint position, this study further verified that the force information can also be decoded by the A-mode ultrasound features. On the other hand, this study demonstrates that A-mode ultrasound is a perfect alternative for EMG signals in human-machine collaboration.

\subsection{Feature space analysis}

Figure 7 shows the first principal component distributions of the ultrasound/sEMG features in terms of elbow angle or torque. The features corresponding to elbow flexion and extension processes were represented in different labels. It clearly indicated that the ultrasound signal features showed more stable mapping relation to angle as well as torque than the sEMG features. For example, as shown in Figure 7 (a) and (b), considering the extension process, the features of EMG were in the obviously centralized distribution in the range from -300 to -200 when the normalized angle $<0.7$, while the ultrasound features were more widely distributed among the whole first principal component range. That resulted in the EMG-based angle prediction with lower accuracy and higher deviation after regression, compared with the ultrasound-based angle prediction. Besides, the features for flexion were separated from the features for extension, which means the angle and torque corresponding to flexion and extension can be distinguishable by the regression model considering all the other principal components.

The sEMG signals are non-stationary signals reflecting muscular activation driven by the moto neuron, which means EMG signal intensity is related to the muscle volunteer contraction degree and insensitive to joint angle. To be specific, during the elbow isokinetic movement, the upper arm muscles were working on maximum voluntary contraction (MVC) at different elbow angle. The patterns for different elbow joint angles were easily confused because of the small differences of sEMG during MVC (e.g. Figure 7(b)), which led to the low regression accuracy in angle prediction (Figure 5(a) and Figure 4(a)). The angle of the elbow together with the MVC during isokinetic movement resulted in the elbow torque, thus, the EMG-related torque prediction accuracy could be higher than the EMG-related angle prediction accuracy (Figure 5(b) and Figure 4(b)), since the angle differences made the torque patterns be more distinguishable (e.g. Figure 7 (d)). That's why the researches in [32][33] applied the highdensity sEMG (more than 16 channels) to increase the sEMG spatial resolution to capture more information in purpose of increasing the angle-related prediction accuracy.

Compared with EMG signals, the ultrasound signals showed higher stability and spatial resolution. On the one hand , studies in [34] had verified the linear relationship between muscle deformation and normalized elbow torque, which indicated that the ultrasound features contain muscle force information; on the other hand, the mapping relationship between elbow angle and upper arm muscle deformation was stable during MVC, and the muscle deformation changed significantly and repeatedly during the elbow isokinetic flexion and extension (as shown in Figure 7 (a) and (c)). So, the ultrasound features, containing the muscle force and deformation information, could better map the angle and torque patterns than the EMG features and increase the prediction accuracy.

\subsection{Future work}

The ultrasound signals are sensitive to the transducer position and detecting direction, which means the low robustness for transducer shift. Besides, only the elbow joint and the 60 degree/second isokinetic movements were involved in this study. Our future work will focus on: 1) improving the ultrasound signal robust against the transducer shift; 2) the biosignals based decoding towards wrist and shoulder joint angle and torque; 3) the bio-signal decoding respect to the isokinetic movements in different angular velocities as well as the isometric contraction in different joint angles.

This work is supported by the National Natural Science Foundation of China (No. 51575338, 51575407, 51475427, 61733011) and the Fundamental Research Funds for the Central Universities (17JCYB03).

1 Ding H, Yang X, Zheng N, et al. Tri-Co Robot: A Chinese Robotic Research Initiative for Enhanced Robot Interaction Capabilities[J]. 2017.

2 Artemiadis P K, Kyriakopoulos K J. An EMG-Based Robot Control Scheme Robust to Time-Varying EMG Signal Features[J]. IEEE Transactions on Information Technology in Biomedicine A Publication of the IEEE Engineering in Medicine \& Biology Society, 2010, 14(3):582-8. 
3 Lambrecht J M, Pulliam C L, Kirsch R F. Virtual reality environment for simulating tasks with a myoelectric prosthesis: an assessment and training tool[J]. Journal of Prosthetics \& Orthotics Jpo, 2011, 23(2):89.

4 Fang Y, Hettiarachchi N, Zhou D, et al. Multi-Modal Sensing Techniques for Interfacing Hand Prostheses: A Review[J]. IEEE Sensors Journal, 2015, 15(11):6065-6076.

5 Parker P, Englehart K, Hudgins B. Myoelectric signal processing for control of powered limb prostheses[J]. J Electromyogr Kinesiol, 2006, 16(6):541-548.

6 Hudgins B, Parker P, Scott R N. A new strategy for multifunction myoelectric control.[J]. IEEE Trans Biomed Eng, 1993, 40(1):82-94.

7 Lucas M F, Gaufriau A, Pascual S, et al. Multi-channel surface EMG classification using support vector machines and signal-based wavelet optimization[J]. Biomedical Signal Processing \& Control, 2008, 3(2):169-174

8 Hargrove L J, Scheme E J, Englehart K B, et al. Multiple Binary Classifications via Linear Discriminant Analysis for Improved Controllability of a Powered Prosthesis[J]. IEEE Trans Neural Syst Rehabil Eng, 2010, 18(1):49-57.

9 Ameri A, Kamavuako E N, Scheme E J, et al. Support vector regression for improved real-time, simultaneous myoelectric control.[J]. IEEE Transactions on Neural Systems \& Rehabilitation Engineering, 2014, 22(6):1198-1209.

10 Hahne J M, Biessmann F, Jiang N, et al. Linear and nonlinear regression techniques for simultaneous and proportional myoelectric control.[J]. IEEE Trans Neural Syst Rehabil Eng, 2014, 22(2):269-279.

11 Hodges P W, Pengel L H, Herbert R D, et al. Measurement of muscle contraction with ultrasound imaging.[J]. Muscle \& Nerve, 2003, 27(6):682.

12 Kiesel K B, Uhl T L, Underwood F B, et al. Measurement of lumbar multifidus muscle contraction with rehabilitative ultrasound imaging[J]. Manual Therapy, 2007, 12(2):161-166.

13 Shi J, Hu S X, Liu Z, et al. Recognition of Finger Flexion from Ultrasound Image with Optical Flow: A Preliminary Study[C]// International Conference on Biomedical Engineering and Computer Science. IEEE, 2010:1-4.

14 Castellini C, Passig G, Zarka E. Using Ultrasound Images of the Forearm to Predict Finger Positions[J]. IEEE Transactions on Neural Systems \& Rehabilitation Engineering, 2013, 20(6):788-797.

15 Sierra G D, Castellini C. A realistic implementation of ultrasound imaging as a human-machine interface for upper-limb amputees[J]. Frontiers in Neurorobotics, 2013, 7(17):17.

16 Sikdar S, Rangwala H, Eastlake E, et al. Novel Method for Predicting Dexterous Individual Finger Movements by Imaging Muscle Activity Using a Wearable Ultrasonic System.[J]. IEEE Transactions on Neural Systems \& Rehabilitation Engineering, 2014, 22(1):69-76.

17 Li Y, He K, Sun X, et al. Human-machine interface based on multichannel single-element ultrasound transducers: A preliminary study[C]// IEEE, International Conference on E-Health Networking, Applications and Services. IEEE, 2016:1-6.

18 Huang Y, Yang X, Li Y, et al. Ultrasound-based Sensing Models for Finger Motion Classification[J]. IEEE Journal of Biomedical \& Health Informatics, 2017, PP(99):1-1.

19 Sun X, Yang X, Zhu X, et al. Explore Dual-frequency Ultrasound Transducers for Morphological Changes of Deep-layered Muscles[J]. IEEE Sensors Journal, 2017, PP(99):1-1.

20 Yang X, Sun X, Zhou D, et al. Towards wearable A-mode ultrasound sensing for real-time finger motion recognition[J]. IEEE Transactions on Neural Systems and Rehabilitation Engineering, 2018.

21 Akhlaghi N, Baker C, Lahlou M, et al. Real-time Classification of Hand Motions using Ultrasound Imaging of Forearm Muscles.[J]. IEEE Trans Biomed Eng, 2016, 63(8):1687-1698.

22 Hislop H J, Perrine J J. The isokinetic concept of exercise[J]. Physical Therapy, 1967, 47(2):114
23 Delitto A, Rose S J, Crandell C E, et al. Reliability of isokinetic measurements of trunk muscle performance.[J]. Spine, 1991, 16(7):800-803.

24 Lucas M F, Gaufriau A, Pascual S, et al. Multi-channel surface EMG classification using support vector machines and signal-based wavelet optimization[J]. Biomedical Signal Processing \& Control, 2008, 3(2):169-174

25 Xie H B, Zheng Y P, Guo J Y, et al. Estimation of wrist angle from sonomyography using support vector machine and artificial neural network models[J]. Medical Engineering \& Physics, 2009, 31(3):384-391.

26 Shuang Q, Jing F, Xu J, et al. Sonomyography Analysis on Thickness of Skeletal Muscle During Dynamic Contraction Induced by Neuromuscular Electrical Stimulation: A Pilot Study[J]. IEEE Trans Neural Syst Rehabil Eng, 2017, 25(1):59-67.

27 Guo J Y, Zheng Y P, Xie H B, et al. Towards the application of onedimensional sonomyography for powered upper-limb prosthetic control using machine learning models[J]. Prosthetics \& Orthotics International, 2013, 37(1):43-49.

28 Hettiarachchi N, Ju Z, Liu H. A New Wearable Ultrasound Muscle Activity Sensing System for Dexterous Prosthetic Control[C]// IEEE International Conference on Systems, Man, and Cybernetics. IEEE, 2016:1415-1420.

29 Szabo T L. Diagnostic Ultrasound Imaging: Inside Out (Second Edition)[M]. 2014.

30 Smola A J, Schölkopf B. A tutorial on support vector regression[J]. Statistics and computing, 2004, 14(3): 199-222.

31 Liu M M, Herzog W, Savelberg H H. Dynamic muscle force predictions from EMG: an artificial neural network approach.[J]. Journal of Electromyography \& Kinesiology, 1999, 9(6):391-400.

32 Tenore F, Ramos A, Fahmy A, et al. Towards the Control of Individual Fingers of a Prosthetic Hand Using Surface EMG Signals[C]// Engineering in Medicine and Biology Society, 2007. Embs 2007. International Conference of the IEEE. IEEE, 2007:6145-6148.

33 Amma C, Krings T, Schultz T. Advancing Muscle-Computer Interfaces with High-Density Electromyography[C]// ACM Conference on $\mathrm{Hu}-$ man Factors in Computing Systems. ACM, 2015:929-938.

34 Shi J, Zheng Y P, Huang Q H, et al. Continuous monitoring of sonomyography, electromyography and torque generated by normal upper arm muscles during isometric contraction: sonomyography assessment for arm muscles[J]. IEEE Trans Biomed Eng, 2008, 55(3):1191-1198. 